\title{
Impact of fermentation, drying, roasting and Dutch processing on flavan-3-ol stereochemistry in cacao beans and cocoa ingredients
}

\author{
W Jeffrey Hurst ${ }^{1 *}$, Susann H Krake ${ }^{2}$, Stephen C Bergmeier ${ }^{2}$, Mark J Payne ${ }^{1}$, Kenneth B Miller ${ }^{1}$ and David A Stuart ${ }^{1}$
}

\begin{abstract}
This paper reports a systematic study of the level of flavan-3-ol monomers during typical processing steps as cacao beans are dried, fermented and roasted and the results of Dutch-processing. Methods have been used that resolve the stereoisomers of epicatechin and catechin. In beans harvested from unripe and ripe cacao pods, we find only (-)-epicatechin and (+)-catechin with (-)-epicatechin being by far the predominant isomer. When beans are fermented there is a large loss of both (-)-epicatechin and (+)-catechin, but also the formation of (-)-catechin. We hypothesize that the heat of fermentation may, in part, be responsible for the formation of this enantiomer. When beans are progressively roasted at conditions described as low, medium and high roast conditions, there is a progressive loss of (-)-epicatechin and (+)-catechin and an increase in (-)-catechin with the higher roast levels. When natural and Dutch-processed cacao powders are analyzed, there is progressive loss of both (-)-epicatechin and (+)-catechin with lesser losses of (-)-catechin. We thus observe that in even lightly Dutch-processed powder, the level of (-)-catechin exceeds the level of (-)-epicatechin. The results indicate that much of the increase in the level of (-)-catechin observed during various processing steps may be the result of heat-related epimerization from (-)-epicatechin. These results are discussed with reference to the reported preferred order of absorption of $(-)$-epicatechin $>(+)$-catechin $>(-)$-catechin. These results are also discussed with respect to the balance that must be struck between the beneficial impact of fermentation and roasting on chocolate flavor and the healthful benefits of chocolate and cocoa powder that result in part from the flavan-3-ol monomers.
\end{abstract}

\section{Introduction}

Dark chocolate and cocoa powders are associated with numerous health benefits, most notably to the improvement of factors impacting cardiovascular health. Evidence comes from epidemiological studies which associate decreased risk of stroke and cardiovascular disease with the long-term consumption of chocolate [1-3]. Further evidence comes from the notable improvements in endothelial function which lead to increased flow mediated dilation $[4,5]$ and increased flexibility of the vascular system, also known as augmentation $[6,7]$. Studies also show that blood pressure decreases after short-term consumption of dark chocolate [8-10]. Additionally chocolate and/or cocoa have been shown to decrease platelet aggregation $[11,12]$ and decreases

\footnotetext{
* Correspondence: whurst@hersheys.com

${ }^{1}$ The Hershey Center for Health and Nutrition, The Hershey Company, 1025 Reese Avenue, Hershey, PA 17033-0805, USA

Full list of author information is available at the end of the article
}

oxidation of low density lipoprotein [13]. All of these responses are associated with improved health in the overall vascular system. When it comes to the heart itself, dark chocolate consumption has been associated with increased dilation [14] and improved blood flow [15] to the left anterior descending coronary artery. These benefits have been summarized in recent review articles $[16,17]$.

The flavan-3-ols in chocolate and cocoa have been implicated in these cardiovascular benefits. Dark chocolates are high in flavan-3-ols $[18,19]$ and natural, nonDutch processed cocoa powders are richer still in these compounds $[20,21]$. Research indicates that the monomeric flavanols, and especially (-)-epicatechin, are at least partly responsible for these effects. Evidence is based on uptake and conjugation of epicatechin and catechin in the blood, with their levels peaking at about 1 to 2 hours postconsumption [22-24]. The levels of epicatechin in blood are also co-incident with the peak in vasodilation seen in 
brachial arteries as measured by flow-mediated dilation. Bioavailability of flavanols appears to be highest for the monomers, (-)-epicatechin and (+)-catechin, with little of the dimeric oligomers and none of the trimers and higher oligomers appearing in the blood stream [24]. Finally it has been shown that the absorption of (-)-epicatechin into the blood stream is greatest followed by $(+)$-catechin and then (-)-catechin [25].

It has long been known that heavy flavanol losses occur when cocoa beans are fermented [26-28]. Losses also occur during roasting [28], Dutch processing $[20,21]$ and baking with alkali-leavening agents [29]. Fresh cacao beans contain about 12.8 to $43.2 \mathrm{mg} / \mathrm{g}$ of (-)-epicatechin, depending on variety [28-31]. Fresh beans also contain about 20 to 30-times less (+)-catechin [31]. A recent paper comprehensively summarized the importance of fermentation, drying, roasting and Dutch processing on the levels of epicatechin and catechin in cacao beans and cocoa ingredients. This report found that epicatechin, compared to catechin, is as much as 30 -times greater in fresh and dried cocoa beans, but as conventional processing occurs, there is a loss in epicatechin and at times an increase in catechin [31].

Two reports have shown that roasting or Dutch processing can result in the formation of a new stereoisomer, $(-)$-catechin, an enantiomer not found in fresh cacao beans $[32,33]$. But the first report by Gotti et al. [32] only had limited quantitative data for unripe and ripe beans and the second report by Kofink et al. [33] only measured ratios of stereoisomers, not their absolute levels. These, and other reports [20,31-34], speculate that the (-)-catechin is a product of epimerization coming from (-)-epicatechin. One paper did not measure the levels of the stereoisomers of either epicatechin or catechin, only measuring total epicatechin and total catechin [35]. However a recent report [34] concluded that (-)-epicatechin epimerizes to (+)-catechin after roasting and Dutch processing. Obviously there is need to quantify the levels of these flavan-3-ol stereoisomers after typical processing steps common to chocolate and cocoa powder manufacture. Additionally it is important to resolve the disparity in the literature regarding epimerization of (-)-epicatechin to either (-)-catechin or to $(+)$-catechin and to resolve this controversy in the literature.

This manuscript describes the first comprehensive study of the impact of fermentation, drying, roasting and alkali(Dutch) processing of cacao beans and cocoa ingredients by measuring the absolute levels of stereoisomers of epicatechin and catechin. The methods sufficiently resolve the $(+)$ and (-) isomers of epicatechin and catechin, enabling us to show that processing with heat and with alkali result in significant loss of both (-)-epicatechin and (+)-catechin as well as the de novo appearance of $(-)$-catechin, not found in fresh plant material, but formed in fermented as well as roasted and Dutch-processed cocoa beans and ingredients.

\section{Materials and methods \\ Experimental}

HPLC-grade standards of (-)-epicatechin, (-)-catechin and $(+)$-catechin hydrate were purchased from Sigma.

Preparation of the chocolate samples followed a procedure, which was modified from the method used by Donovan et al. in 2006 to observe the bioavailability in chocolate samples [25]. Powdered samples $(2 \mathrm{~g})$ were extracted with $3 \times 4.5 \mathrm{~mL}$ hexanes and the residue was dried under an argon stream overnight. The defatted and dry solid was further extracted with $3 \times 2.5 \mathrm{~mL}$ acetone: $\mathrm{H}_{2} \mathrm{O}: \mathrm{AcOH}$ (70:28:2, v:v:v). The combined extracts were concentrated to approximately $2 \mathrm{~mL}$ under a stream of argon over $36 \mathrm{~h}$. The solution was diluted to exactly $5 \mathrm{~mL}$ with a mixture of acetone: $\mathrm{H}_{2} \mathrm{O}$ : $\mathrm{CH}_{3} \mathrm{CN}(2: 2: 1$, v:v:v).

The analysis was performed on a Shimadzu HPLC with a Astec Cyclobond I 2000 RSP, $5 \mu \mathrm{m}, 250 \times 4.6 \mathrm{~mm}$ column (Supelco Analytical). Two solvents with a gradient were used as mobile phase with a flow rate of $1 \mathrm{~mL} / \mathrm{min}$. Solvent A contained $50 \mathrm{mM}$ aqueous $\mathrm{NaH}_{2} \mathrm{PO}_{4}$ at a pH of 3.0 and solvent $\mathrm{B}$ contained $30 \mathrm{mM}$ aqueous $\mathrm{NaH}_{2} \mathrm{PO}_{4}$ at pH 3.0 in $80 \%$ acetonitrile. A linear gradient from 10.0 to $13.5 \% \mathrm{~B}$ over $45 \mathrm{~min}$, increasing to $45 \% \mathrm{~B}$ at $70 \mathrm{~min}$ and $90 \% \mathrm{~B}$ at $72 \mathrm{~min}$ was applied. $4 \mu \mathrm{L}$ of the samples were injected and a UV-Vis-detector at $214 \mathrm{~nm}$ was utilized for detection. Figure 1 provides an example chromatogram showing the separation of the various epicatechin and catechin sterioisomers while Figure 2 provides a chromatogram showing the stereoisomer separation on sample of roasted cocoa beans. Structures of the various isomers can be see in Figure 3.

\section{Samples}

\section{Fresh Beans}

Fresh cacao pods, both unripe and ripe, of the Forestaro type were obtained from the U.S.D.A. Field Station, Mayaguez, Puerto Rico. Unripe pods were green and the pulp was a spongy white foam typical of unripe pods. Ripe pods were yellow and/or orange and the pulp was moist and watery, typical of ripe pods.

\section{Dried Cacao Beans}

Unfermented washed and dried beans, sometimes referred to as cacao lavado, were commercially obtained from AMSA in Mexico. Cacao beans with various degrees of fermentation from Sulawesi, Ivory Coast and Papua New Guinea (PNG) were commercially available, fair to average quality beans typical of the origin. Commercial beans are nominally $\leq 8 \%$ moisture.

Natural and Dutch-processed (alkalized) cocoa powders were obtained from J.B. Cocoa. Natural cocoa 


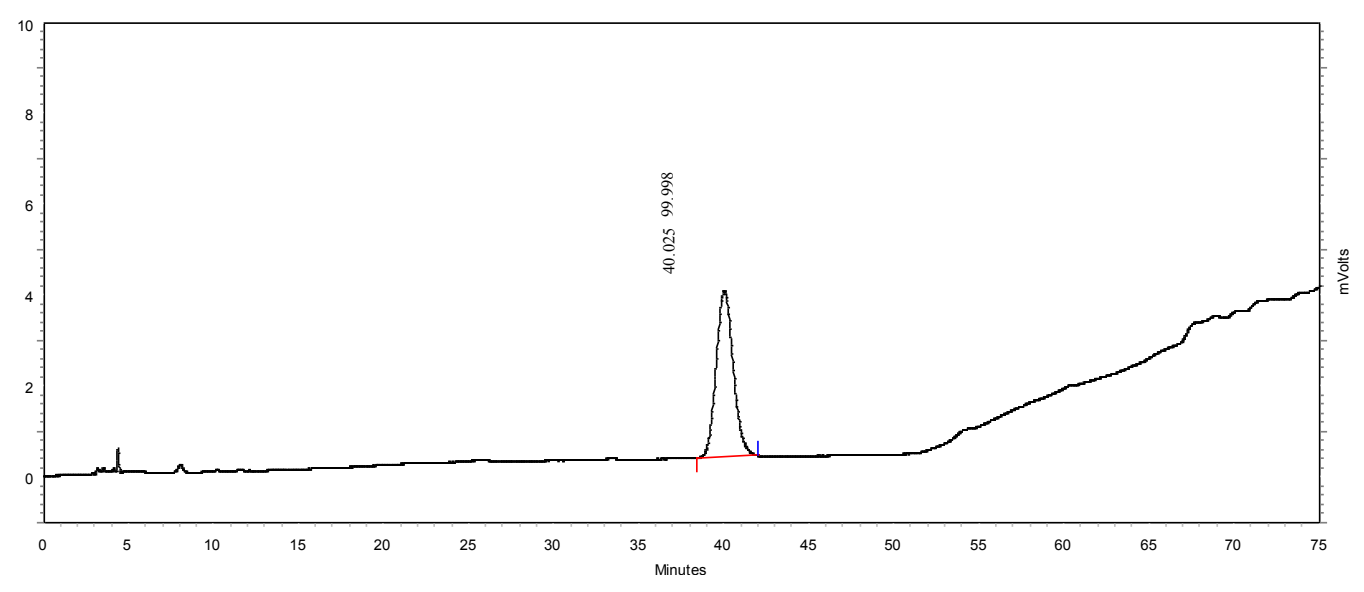

$(+)$-Catechin hy drate

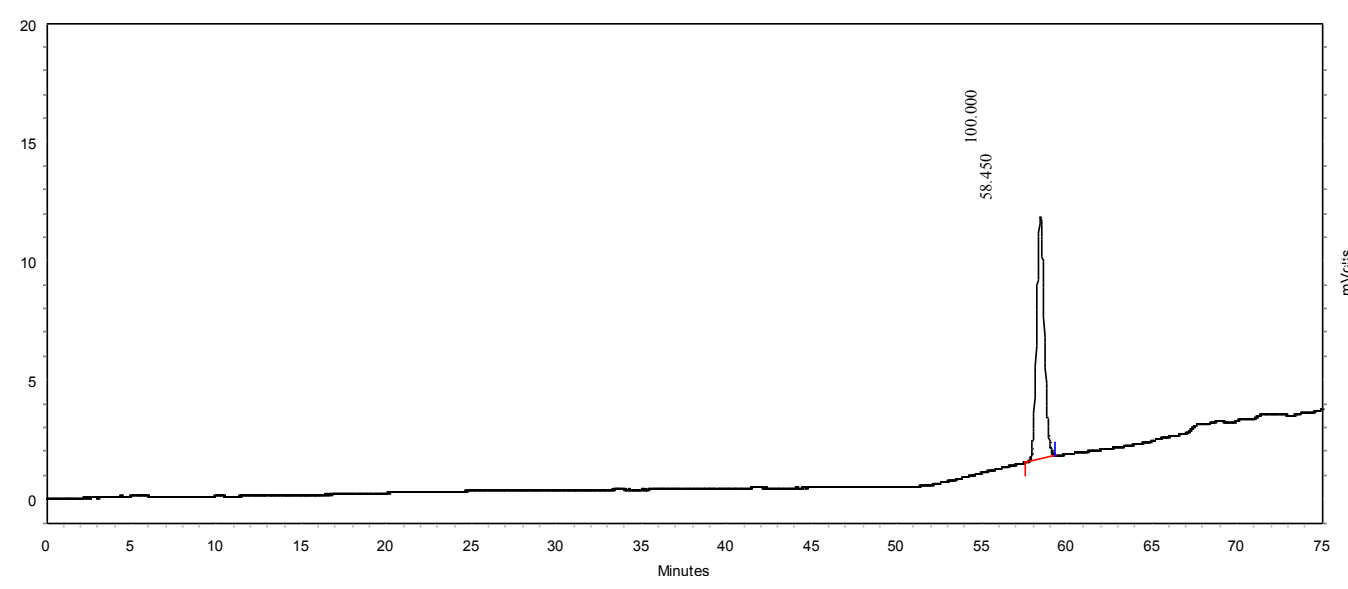

(-)-Catechin

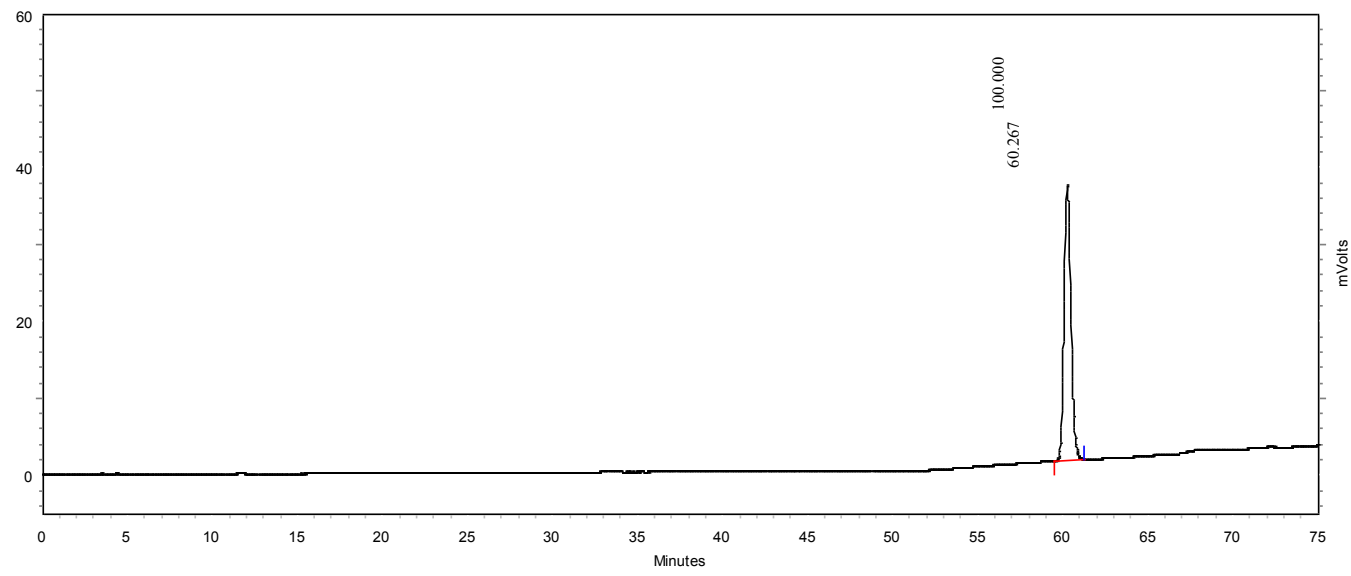

Figure 1 Sample chromatogram of standard compounds. 
sample 33



sample 33

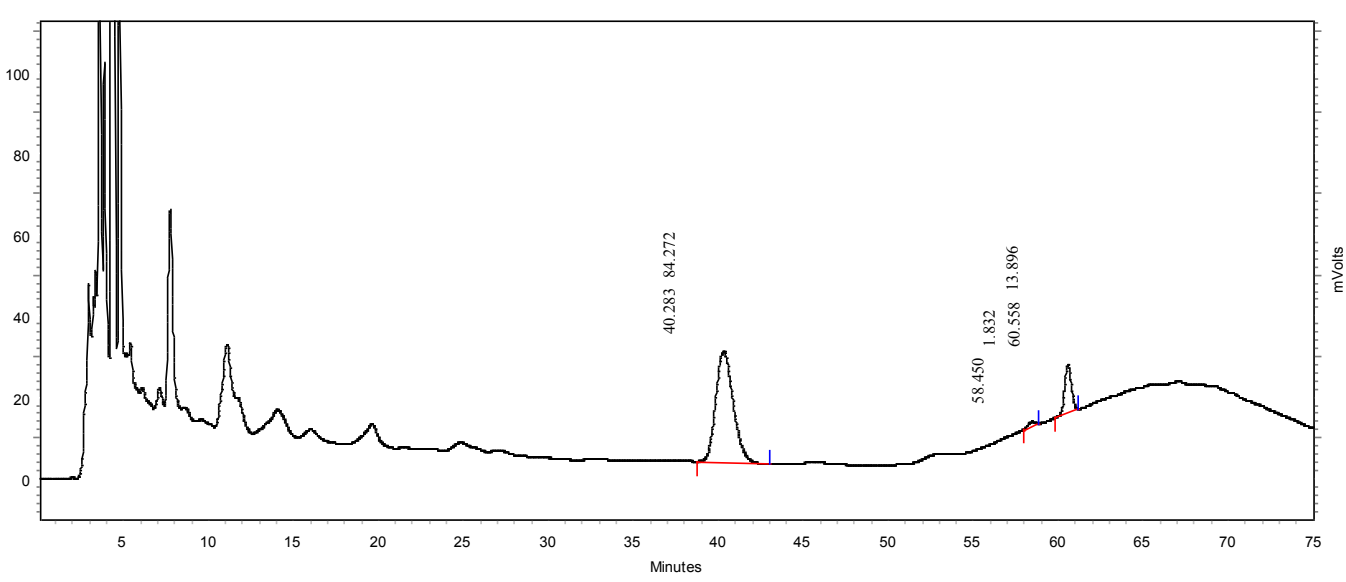

sample 33

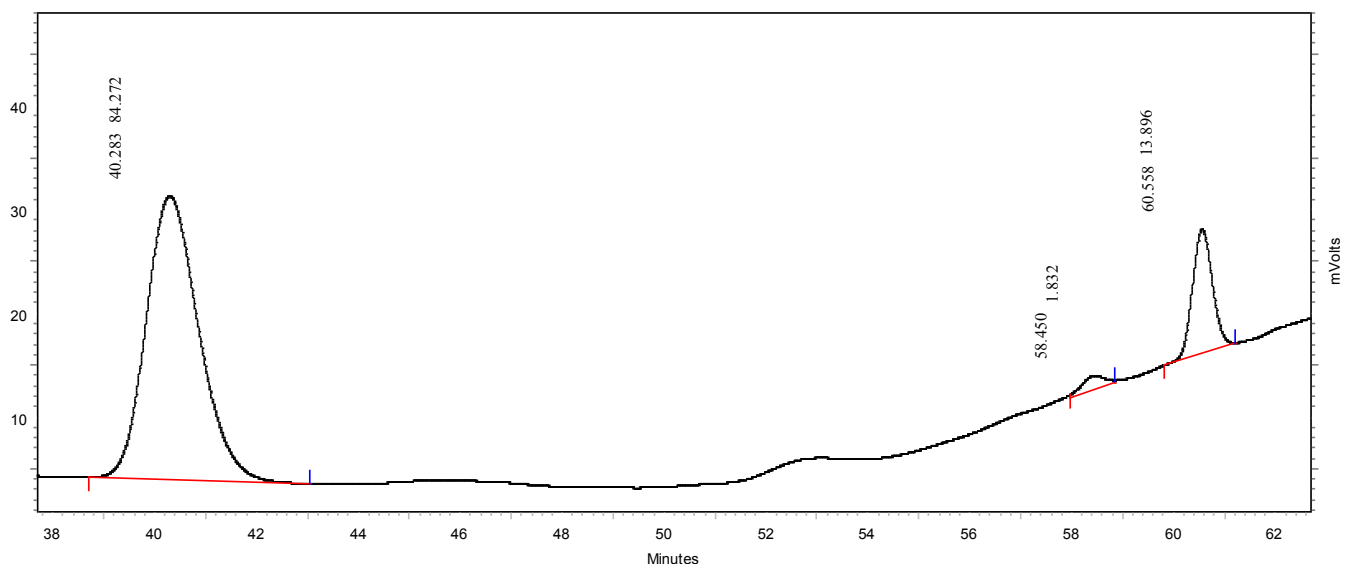

Figure 2 Sample chromatogram of cocoa bean extract. 
<smiles>Oc1cc(O)c2c(c1)O[C@H](c1ccc(O)c(O)c1)[C@H](O)C2</smiles>

(+)-catechin<smiles>Oc1cc(O)c2c(c1)O[C@H](c1ccc(O)c(O)c1)[C@H](O)C2</smiles>

(-)-catechin

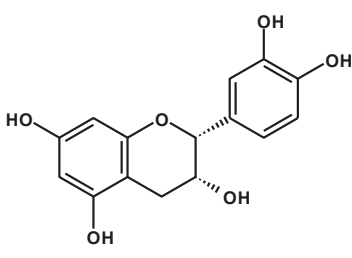

(-)-epicatechin

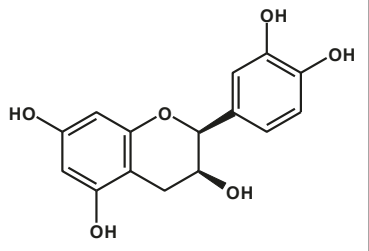

(+)-epicatechin
Figure 3 Structures of (+)-catechin, (-)-epicatechin, (-)-catechin and (+)-epicatechin.

powder represents no Dutch processing with an average extractable $\mathrm{pH}$ of 5.76 while low, medium, and heavy Dutch processed samples were characterized by increasing $\mathrm{pH}$ values of $7.13,7.36$, and 7.92 respectively as described in Miller et al. 2008 [21].

\section{Sample Preparation}

\section{Fresh Beans}

Cacao beans from either unripe or ripe pods were removed within three days of harvest, hand dissected using a scalpel, and freed from the surrounding, foamy pulp (in the case of unripe beans) or watery pulp (in the case of ripe beans). The seed coat was cut away leaving the cotyledons, hypocotyl axis and root radicle. Beans from unripe and ripe pods were freeze-dried to moisture levels at or below $5 \%$.

\section{Roasted Beans}

Commercial grade cacao beans from Ivory Coast were subjected to increasing degrees of roasting using a Blodget Oven. Three hundred grams (300 g) of shell-free cacao nibs were placed on wire mesh trays and roasted in the oven maintained at $163^{\circ} \mathrm{C}$. Low, medium, and high roasted nibs were roasted at 13,20 , and 25 minutes respectively.

\section{Methods}

\section{Degree of Fermentation}

The degree of fermentation of cacao beans was evaluated by acidic methanol extraction and colorimetric determination using a ratio of $460 / 530 \mathrm{~nm}$ based on method of Gourieva and Tserevitinov [36]. The calculated fermentation index values of the three bean samples were: Mexican Lavado (0.5), Sulawesi (0.79), Ivory Coast (0.96), and PNG (1.38).

\section{Results and discussion}

\section{Tabular summary of results}

Table 1 shows representative results for the measurement of (-)-epicatechin, (+)-catechin and (-)-catechin in cacao beans and cocoa ingredients. Note first that unripe and ripe beans contain no detectable amounts of (-)-catechin. This is in agreement with previous findings regarding the occurrence of only (-)-epicatechin and $(+)$-catechin in plants [20,21,26-31]. Further notice that as cacao beans were heated during drying, fermentation, roasting and after Dutch processing, there is a progressive loss of (-)-epicatechin. This loss has been observed for total epicatechin [30,31] and for resolved (-)-epicatechin [32,33]. There is also the loss of $(+)$-catechin in cacao beans or cocoa powder with progressive processing. Finally, note that as beans undergo heating due to fermentation, roasting or Dutch processing, there is the appearance of $(-)$-catechin, which is the dominant form of the catechin present after cacao beans have been Dutch-processed to cocoa powder.

\section{Comparison of data to other literature results}

Also in Table 1 for comparative purposes, data from Payne et al. [31] is shown in which the method of Nelson and Singletary [35] was used to determine total epicatechin and total catechin in unripe, ripe dried, fermented cacao beans and in Dutch processed cocoa powder. The levels of total epicatechin in closely, but not perfectly, correspond to the levels of (-)-epicatechin seen in this study. This difference probably represents the natural variation of cacao beans and powder samples rather than a systematic error in either of the analytical procedures. The same can be said for the amounts of total catechin icompared to the amount of $(+)$ - and $(-)$-catechin summed as total catechin. We conclude that, within the natural variation of cacao ingredients surveyed and sample to sample differences, the findings we have reported for the resolved isomers of (-)-epicatechin, (+)-catechin and (-)-catechin closely agree with the results found previously for total epicatechin and total catechin in similarly processed samples [29]. Our results for the amounts of catechin isomers are also in agreement with the amounts found in Venezuelan cocoa beans from unripe pods by Gotti et al. [33] who measured $15.3 \pm 0.09 \mathrm{mg} / \mathrm{g}(-)$-epicatechin and $(+)-0.45 \pm$ $0.01 \mathrm{mg}$ of $(+)$-catechin.

\section{Analysis of unripe, ripe cacao beans}

In Table 1 is shown the results for the analysis of flavan-3-ols stereoisomers in cacao beans that were unripe and ripe and were subsequently freeze dried. Fresh ripe cacao beans have the highest levels of $(-)$-epicatechin $(13.35 \pm 2.24 \mathrm{mg} / \mathrm{g})$ with 24 -times less $(+)$-catechin 
Table 1 Comparison of data for determination of flavanol stereo-isomer levels of triplicate samples of beans and powder analyzed in this study versus data from an earlier study where only catechin and epicatechin were determined

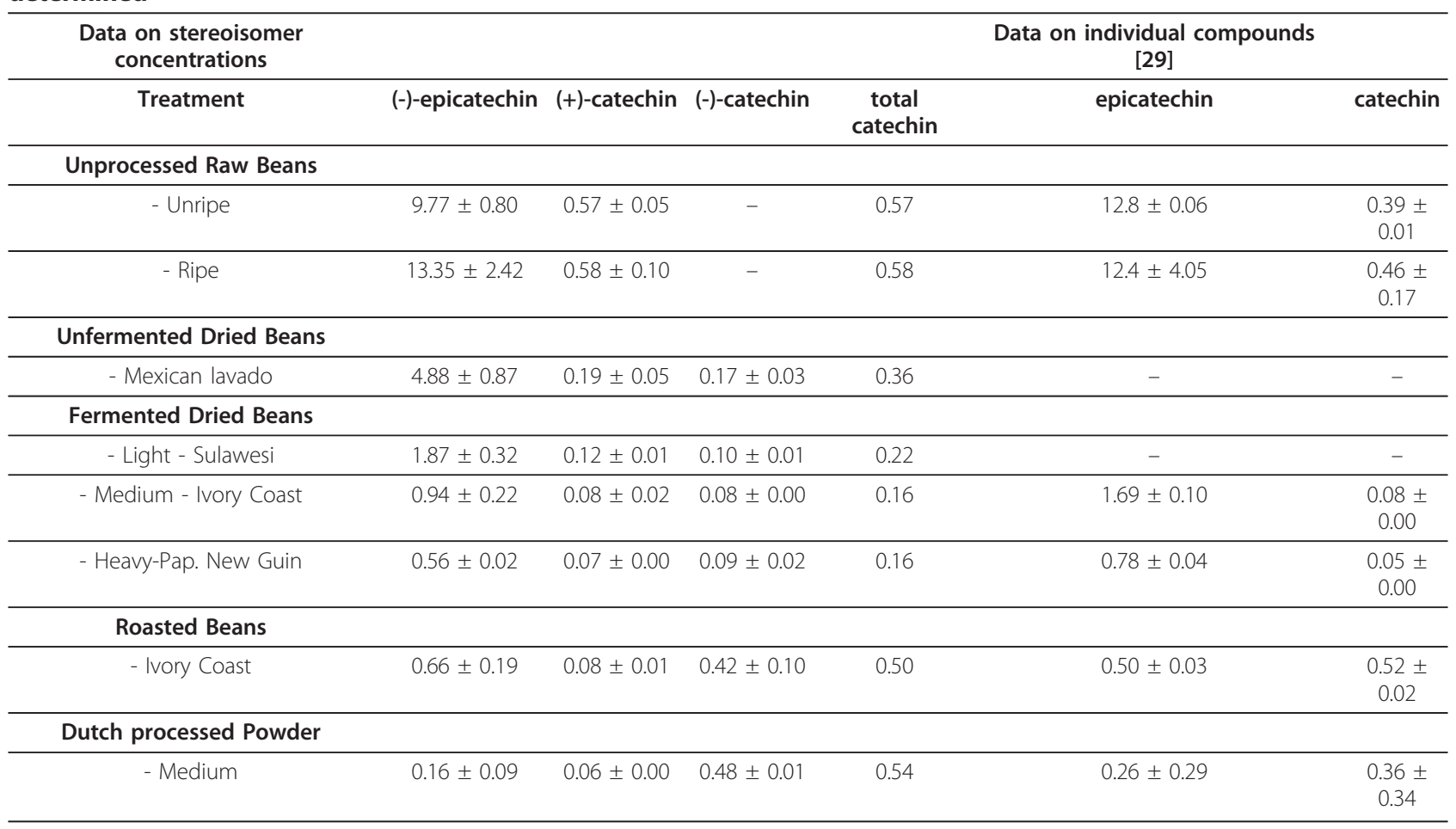

All data are shown on a dried fresh weight basis. For cocoa powders, see Material and Methods for correction factors calculations. All values are in mg/g \pm standard deviation.

A Samples purchased and analyzed in 2004 and published in Miller et al. 2006 [18].

$(0.56 \pm 0.05 \mathrm{mg} / \mathrm{g})$ and no $(-)$-catechin. Unripe beans also have high levels of $(-)$-epicatechin $(9.77 \pm 0.80 \mathrm{mg} /$ g) but somewhat lower than that of ripe beans. The $(+)$-catechin level $(0.57 \pm 0.05 \mathrm{mg} / \mathrm{g})$ of unripe beans is the same as ripe beans.

\section{Effect of cacao bean fermentation}

The results for ripe, freeze dried cacao beans (shown here for comparison), for field processed lavado cacao beans that were sun dried and for cacao beans that had undergone various amounts for intentional fermentation are shown in Figure 4. Lavado beans are beans that have been removed from pods, hand washed in water (lavar in Spanish means "to wash") to remove the surrounding pulp and sun dried. This is a traditional process practiced by native peoples from Southern Mexico, Mesoamerica, Venezuela and the Dominican Republic. While lavado cacao beans are sometimes considered fresh ripe beans, these beans often undergo incidental fermentation. For sun dried, unfermented lavado beans there was a decrease in levels of (-)-epicatechin $(4.26 \pm 0.16 \mathrm{mg} / \mathrm{g})$ and $(+)$-catechin $(0.16 \pm 0.05)$ and the appearance of $(-)$-catechin $(0.15 \pm 0.03 \mathrm{mg} / \mathrm{g})$ compared to ripe, freeze dried beans ((-)-epicatechin, $13.35 \pm 2.42 \mathrm{mg} / \mathrm{g}$; (+)-catechin, $0.56 \pm 0.10 \mathrm{mg} / \mathrm{g})$. This result suggests that the lavado beans have undergone some incidental fermentation during the slow sun-drying process and may have been exposed to heat of fermentation and drying may have caused some loss of (-)-epicatechin and $(+)$-catechin. Heating during incidental fermentation also may be the reason why (-)-catechin appears in dried beans studied here. Throughout the remainder of

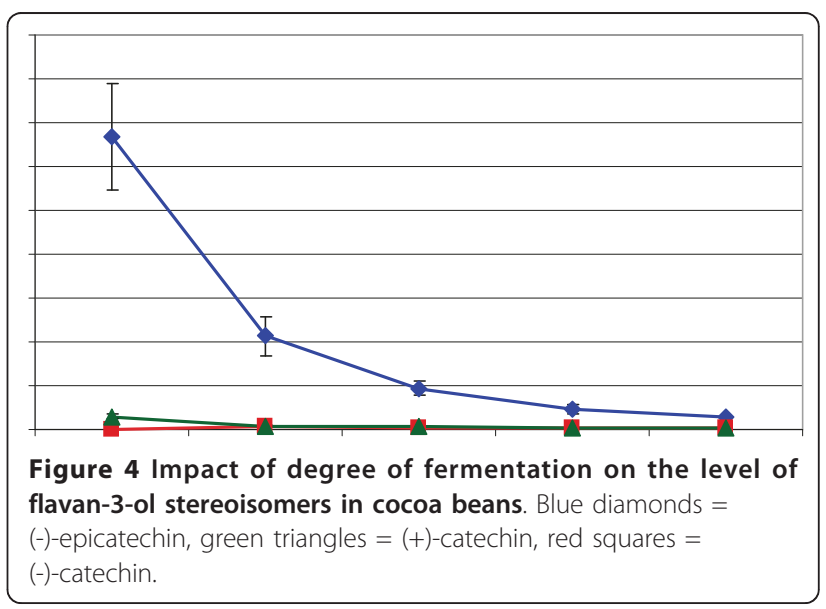


the report ripe, frozen dried beans will be the reference bean at $100 \%$ for the comparison of beans that have been fermented or roasted.

The results for lightly fermented cacao beans, as represented by commercially available Sulawesi cacao beans, are compared to medium fermentation, as represented by Ivory Coast beans, and heavy fermentation, as represented by Papua New Guinea beans, are also shown in Figure 4. Sulawesi has a tradition of either not fermenting or fermenting for a short period, Ivory Coast has a tradition of fermenting for four to five days and Papua New Guinea has a tradition of fermenting for six or more days, although in the beans that were analyzed the absolute length of fermentation was not truly known to us. Compared to dried, unfermented beans, the light fermented Sulawesi beans had an average of $1.87 \pm 0.32$ $\mathrm{mg} / \mathrm{g}$ of $(-)$-epicatechin, $0.12 \pm 0.01 \mathrm{mg} / \mathrm{g}(+)$-catechin and $0.10 \pm 0.01 \mathrm{mg} / \mathrm{g}$ of $(-)$-catechin. The Ivory Coast beans had an average of $0.94 \pm 0.22 \mathrm{mg} / \mathrm{g}$ of (-)-epicatechin, $0.08 \pm 0.01 \mathrm{mg} / \mathrm{g}(+)$-catechin and $0.08 \pm 0.00 \mathrm{mg} /$ $\mathrm{g}$ of (-)-catechin. The heaviest fermented beans, the Papua New Guinea beans, had the least (-)-epicatechin $(0.56 \pm 0.02 \mathrm{mg} / \mathrm{g}),(+)$-catechin $(0.07 \pm 0.00 \mathrm{mg} / \mathrm{g})$ and $(-)$-catechin $(0.09 \pm 0.00 \mathrm{mg} / \mathrm{g})$. Extending the fermentation time decreased the level of (-)-epicatechin and $(+)$-catechin from levels found in ripe and dried beans but also caused a relative increase in (-)-catechin, compared to the other stereoisomers. We interpret this to mean that the temperature of the fermentation mass could be responsible for the appearance of (-)-catechin during fermentation since it has been observed that fermentation temperatures as high as $60^{\circ} \mathrm{C}$ for as long as two days can occur [37].

\section{Effect of roasting on flavan-3-ol stereoisomers}

The impact of roasting for varying lengths of time on Ivory Coast beans from the same lot is shown in Figure 5 . Beans that have undergone low roasting have numerically higher $(1.17 \pm 0.04 \mathrm{mg} / \mathrm{g})$, but not significantly greater amounts of (-)-epicatechin levels compared to unroasted beans $(0.94 \pm 0.22 \mathrm{mg} / \mathrm{g})$. By comparison, the amount of $(-)$-catechin in low roasted beans $(0.31 \pm 0.01$ $\mathrm{mg} / \mathrm{g})$ is higher than in unroasted beans $(0.08 \pm 0.00$ $\mathrm{mg} / \mathrm{g})$. The level of $(+)$-catechin in low roasted beans $(0.08 \pm 0.00 \mathrm{mg} / \mathrm{g})$ is the same as in unroasted beans $(0.08 \pm 0.01 \mathrm{mg} / \mathrm{g})$. There is a trend for the level of (-)-epicatechin to decrease as the degree of roast level increases so that in the lowest and highest roasted beans, the level of (-)-epicatechin decreased to $0.66 \pm$ $0.02 \mathrm{mg} / \mathrm{g}$. Conversely, as roast level increased for the highest roast the amount of $(-)$-catechin $(0.58 \pm 0.02$ $\mathrm{mg} / \mathrm{g})$. The level of $(+)$-catechin decreases only slightly to $0.06 \pm 0.00 \mathrm{mg} / \mathrm{g}$ in high roast beans compared to $0.08 \pm 0.01 \mathrm{mg} / \mathrm{g}$ in unroasted beans.

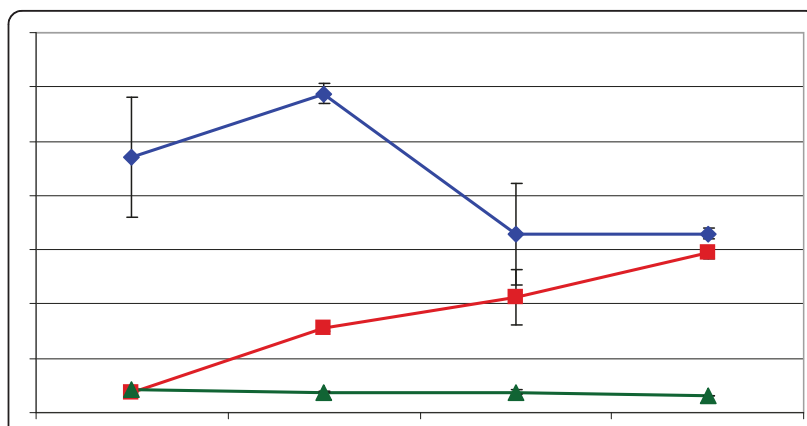

Figure 5 Impact of roasting on the flavan-3-ol stereoisomer content of ivory coast cocoa beans. Blue diamonds =

$(-)$-epicatechin, green triangles $=(+)$-catechin, red squares $=$ $(-)$-catechin

In Figure 6 is shown the analysis of cocoa powders made by pressing the fat out of roasted beans, also known as natural powders, as well as a series of Dutch processed (alkalized) powders, which are described by the manufacturer as light-, medium- and heavily-Dutch processed. Dutch-processing involves treating cocoa beans, nibs, liquor, or powder with alkali and heat. The results show that as the amount of Dutch-processing increases, the amount of (-)-epicatechin, $(+)$-catechin and (-)-catechin all decrease. The results also show that $(-)$-catechin is the predominant flavan-3-ol even in the light Dutch processing, exceeding the level of (-)-epicatechin in the Dutch-processed powders. Data reported by Donovan et al. [25] indicate that the bioavailability of flavan-3-ol monomers in order from high to low availability is $(-)$-epicatechin $>(+)$-catechin $>(-)$-catechin. Our compositional analysis of cocoa powders suggests that A) natural cocoa powder has the highest level of bioavailable flavanols since (-)-epicatechin is in the highest levels followed by (-)-catechin and B) even in lightly Dutch-processed cocoa powders, (-)-catechin is the predominant flavanol antioxidant, exceeding the level of

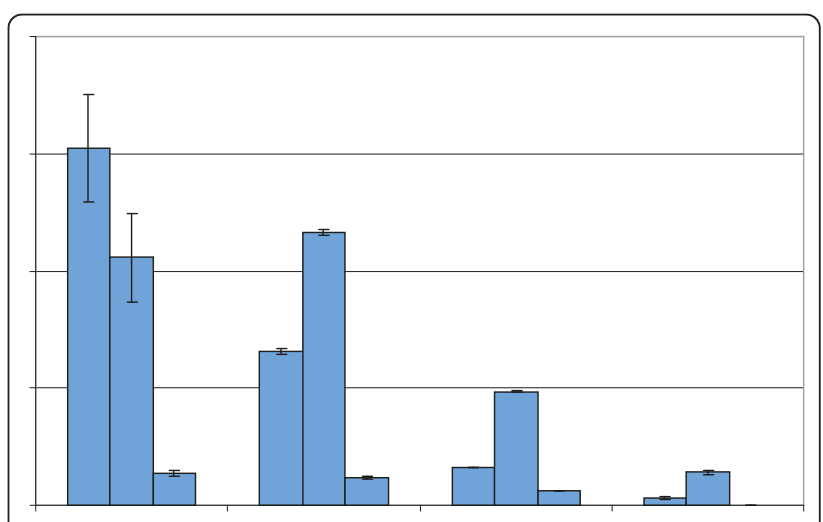

Figure 6 Impact of dutch (alkali) processing on the flavan-3-ol content of cocoa powders. Blue solid bars $=(-)$-epicatechin, green, cross hatched bars $=(+)$-catechin, red diamond bars $=(-)$-catechin. 
(-)-epicatechin. This suggests that even light Dutch processing will have a negative impact on the healthful properties of flavanols due to the relatively high amounts of (-)-catechin, the least bioavailable flavan-3ol (Figure 6).

In Figure 7 is shown a summary of the key bean processing steps and cocoa ingredients described in this report. The weights of the Dutch-processed cocoa powder have been corrected to account for pressing of the fat from the beans (see Materials and Methods). Fresh beans contain about $1.3 \%$ of their weight as (-)-epicatechin. As beans are dried, there is a loss of both (-)-epicatechin and $(+)$-catechin and the appearance of measurable amounts of (-)-catechin. We interpret this to mean that heating during sun-drying and incidental mild fermentation may give rise to the (-)-catechin since we were unable to detect any of this enantiomer in unripe (Figure 4) or ripe beans. Medium fermentation, as defined here as Ivory Coast beans, causes a continued reduction of the level of (-)-epicatechin in the beans which is consistent with the results of a number of reports where there was no separation of the (-) and the $(+)$ enantiomer of epicatechin $[25-27,29]$ and with the report of Kofink et al. [33] who could resolve the (-) and the $(+)$ enantiomers. Roasting beans to a medium

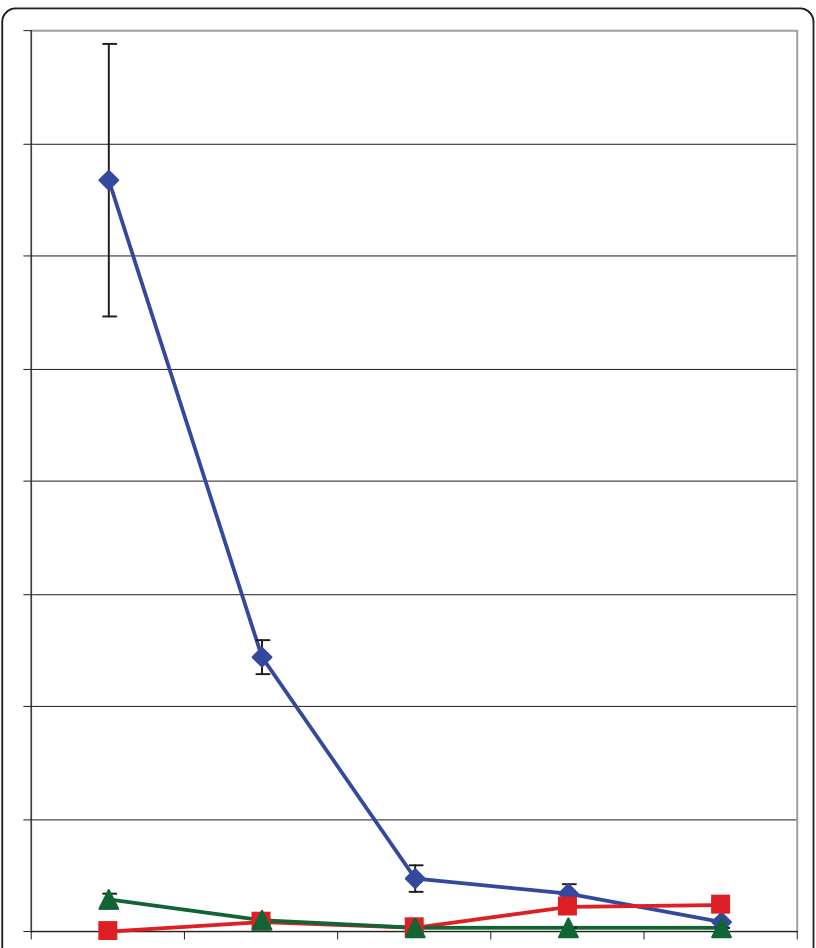

Figure 7 Summary graph of the impact of processing on the flavan-3-ol content of cocoa beans. with exception of the ripe beans, all samples were commercial samples representative of the indicated processing stage. Blue diamonds $=(-)$-epicatechin, green triangles $=(+)$-catechin, red squares $=(-)$-catechin level causes a small loss in (-)-epicatechin but a large increase in (-)-catechin, presumably due to epimerization due to the temperature of roasting. Finally, with medium Dutch processing, one sees the loss of (-)-epicatechin from cocoa powder and the increase in $(-)$-catechin. In Dutched powders, (-)-catechin is the dominant flavan-3-ol monomer. The results shown here are consistent with the results shown by Kofink et al. [33] that suggested that there is epimerization of (-)-epicatechin to (-)-catechin due to the heat of roasting and during Dutch processing of cocoa. Our results show that the absolute level of $(+)$-catechin in unripe and ripe beans is less than the level of $(-)$-catechin in medium Dutch processed beans. Our finding of (-)-catechin differs significantly from those of Caligiani et al. [34] who indicated that (-)-epicatechin is epimerized to (+)-epicatechin; we found no $(+)$-epicatechin in any of the samples tested.

Balance between chocolate palatability and healthfulness As pointed out in the introduction, the association of the cardiovascular benefits of chocolate and cocoa with the flavanols is supported by an ever growing body of literature. So, in order to maximize the impact of cocoa or dark chocolate on health, why not make these products out of unfermented beans? Indeed this concept is the basis of several unique ingredients or chocolates $[38,39]$. But there are good reasons why fermented cocoa beans are used, which mostly concern taste and palatability. One of the quality measures used to determine the final price of cocoa beans is the "cut test". This is a measure of the oxidation of the anthocyanins in the cocoa beans; grey and purple beans being unfermented and brown beans being fully fermented [40,41]. Chocolate makers know that optimally fermented cocoa beans will be less astringent, presumably due to the reduction of flavanols, and these beans also will have more cacao flavor, presumably due to chocolate flavor precursors that, when roasted, produce cacao flavor. So a balance needs to be struck between retaining the health benefits associated with the flavanol antioxidants, especially (-)-epicatechin, and the beneficial impact that fermentation has on roasted flavor and to mitigate excessive astringency. Similar logic holds for roasting. Roasting develops chocolate flavor and aroma, a fact well known to the Mayans who roasted on traditional charcoal heated comals and to anyone who has roasted cocoa beans, whether it is done industrially or in a skillet $[40,41]$. In addition to flavor development, roasting also loosens the cocoa shell, making it easier to remove from the cotyledons of the cocoa bean. Roasting also is a critical pasteurization or even a sterilization step that increases the microbiological safety of the resulting roasted beans, chocolate liquor or cocoa powder. A 
balance needs to be struck between preserving the healthful benefits associated with the flavanol antioxidants, especially (-)-epicatechin, and the loss that occurs during roasting and flavor development that may be key to certain chocolate brand identity.

Cocoa powder and dark chocolate are appreciated, first and foremost, for their flavor and texture. By attempting to make cocoa ingredients healthier by shortening fermentation time or minimizing roasting for similar, we would expect that the result would be a bitter, astringent ingredient with lower than expected cocoa flavor. So as with many things, a balance needs to be struck between different benefits; in this case flavor versus healthfulness.

\section{Acknowledgements}

The project was funded by the Hershey Center for Health \& Nutrition

\section{Author details}

${ }^{1}$ The Hershey Center for Health and Nutrition, The Hershey Company, 1025 Reese Avenue, Hershey, PA 17033-0805, USA. ${ }^{2}$ Department of Chemistry and Biochemistry, Ohio University, Athens, $\mathrm{OH}$ 45701, USA.

\section{Authors' contributions}

SHK extracted samples and performed the analysis with SCB collecting, reviewing and organizing data. WJH designed the experiment and prepared the final paper. MJP performed supplemental analysis and contributed to writing the manuscript, KBM collected samples, developed the code and contributed to the preparation of the manuscript with DAS directing research and preparing the draft manuscript. All authors have read and approved the final manuscript.

\section{Competing interests}

The authors declare that they have no competing interests. SHK and SCB are affiliated with Ohio University. The remainder of the authors are with The Hershey Company

Received: 25 April 2011 Accepted: 14 September 2011

Published: 14 September 2011

\section{References}

1. Buijsse B, Feskens EJ, Kok FJ, Kromhout D: Cocoa intake, blood pressure and cardiovascular mortality: The Zutphen elderly study. Arch Intern Med 2006, 166:411-417

2. Mink PJ, Scrafford CG, Barraj LM, Harnack L, Hong CP, Nettleton JA, Jacobs DRJ: Flavonoid intake and cardiovascular disease mortality: a prospective study in post menopausal women. Am J Clin Nutr 2007, 85:895-909.

3. Buijsse B, Weikert C, Drogan D, Bergmann M, Boeing H: Chocolate consumption in relation to blood pressure and risk of cardiovascular disease in German adults. Eur Heart J 2010, 10:1093-1101.

4. Heiss C, Dejam A, Kleinbongard P, Schewe T, Seis H, Kelm M: Vascular effects of cocoa rich in flavan-3-ols. J Amer Med Assoc 2003, 290:1030-1031

5. Engler MB, Engler MM, Chen CY, Malloy MJ, Browne A, Elisa BS, Chiu Y, Kwak H-K, Milbury P, Paul SM, Blumberg J, Mietus-Snyder ML: Flavonoid rich dark chocolate improves endothelial function and increases epicatechin concentrations in healthy adults. J Am Coll Nutr 2004, 23:197-204.

6. Fischer ND, Hughes M, Gerhard-Herman M, Hollenberg NK: Flavanol-rich cocoa induces nitric oxide-dependent vasodilation in healthy humans. $J$ Hypertension 2003, 21:2281-2286.

7. Vlachopoulos C, Aznaouridis K, Alexopoulos N, Economou E, Andreadou I, Stefanadis C: Effect of dark chocolate on arterial function in healthy individuals. Am J Hypertension 2005, 18:785-791.
8. Taubert D, Berkels R, Roesen R, Klaus W: Chocolate and blood pressure in elderly individuals with isolated systolic hypertension. J Amer Med Assoc 2003, 290:1029-1030.

9. Grassi D, Necozione S, Lippi C, Croce G, Valeri L, Pasqualetti P, Desideri G, Blumberg J, Ferri C: Cocoa reduces blood pressure and insulin resistance and improves endothelial-dependent vasodilation in hypertensives. Hypertension 2005, 46:398-405.

10. Taubert D, Roesen R, Lehmann C, Jung N, Schomig E: Effects of low habitual cocoa intake on blood pressure and bioactive nitric oxide: $\mathrm{A}$ randomized controlled trial. J Amer Med Assoc 2007, 298:49-60.

11. Heiss C, Dejam A, Kleinbongard P, Schewe T, Seis H, Kelm M: Vascular effects of cocoa rich in flavan-3-ols. J Amer Med Assoc 2003, 290:1030-1031.

12. Murphy KJ, Chronopolos AK, Singh I, Francis MA, Moriarty H, Pike MJ, Turner AH, Mann NJ, Sinclair AJ: Dietary flavanols and procyanidin oligomers from cocoa (Theobroma cacao) inhibit platelet function. Am J Clin Nutr 2003, 77:1466-73.

13. Kondo K, Hirano R, Matsumoto A, Igarashi O, Itakura H: Inhibition of LDL oxidantion by cocoa. Lancet 1996, 348:1514.

14. Flammer AJ, Hermann F, Sudano I, Spieker L, Hermann M, Cooper KA, Serafini M, Luscher TF, Ruschitzka F, Noll G, Corti R: Dark chocolate improves coronary vasomotion and reduces platelet reactivity. Circulation 2007, 116:2376-2382.

15. Shiina $Y$, Funabashi N, Lee K, Murayama T, Nakamura K, Wakatsuki $Y$, Daimon M, Kumoro I: Acute effect of oral flavoniod-rich dark chocolate intake on coronary circulation, as compared with non-flavonoid white chocolate, by transthorasic Doppler echocardiography in healthy adults. Int J Cardiol 2009, 131:424-429.

16. Cooper KA, Donovan JL, Waterhouse AL, Williamson G: Cocoa and Health: A decade of Research. Brit J Nutr 2008, 99:1-11.

17. Corti R, Flammer AJ, Hollenberg NK, Luscher TF: Cocoa and Cardiovascular Health. Circulation 2009, 119:1433-41.

18. Miller KB, Stuart DA, Smith NL, Lee CY, Mchale NL, Flanagan JA, Ou B, Hurst WJ: Antioxidant activity and polyphenol and procyanidins contents of selected commercially available cocoa-containing and chocolate products in the United States. J Agric Food Chem 2006, 54:4062-68.

19. Cooper KA, Campos-Gimenez E, Alverez DJ, Nagy K, Donovan JA, Williamson GA: Rapid reversed phase ultra-performance liquid chromatography analysis of the major cocoa polyphenols and interrelationships of their concentrations in chocolate. J Agric Food Chem 2007, 56:260-265.

20. Andres-Lacueva C, Monagas M, Khan N, Izquierdo-Pulido M, Urpi-Sarda M, Permanyer J, Lamuela-Raventtos RM: Flavanol and Flavonol contents of cocoa powder products: Influence of manufacturing process. J Agric Food Chem 2008, 56:3111-17.

21. Miller KB, Hurst WJ, Payne MJ, Stuart DA, Apgar J, Sweigart DS, Ou B: Impact of alkalization on the antioxidant and flavanol content of commercial cocoa powders. J Agric Food Chem 2008, 56:8527-8533.

22. Richelle M, Tavazzi I, Enslen M, Offord EA: Plasma kinetics in man of epicatechin from dark chocolate. Eur J Clin Nutr 1999, 53:22-26.

23. Schroeter H, Heiss C, Balzer J, Kleinbongard P, Keen CL, Hollenberg NK, Sies H, Kwik-Uribe C, Schmitz HH, Kelm M: (-)-Epicatechin mediates beneficial effects of flavanol-rich cocoa on vascular function in humans. P Nat Acad Sci USA 2006, 103:1024-1029.

24. Holt RR, Lazarus SA, Sullards MC, Zhu QY, Schramm DD, Hammerstone JF, Frage CG, Schmitz HH, Keen CL: Procyanidin dimer B2 [epicatechin-4beta8)epicatechin] in human plasma after consumption of a flavanol-rich cocoa. Am J Clin Nutr 2002, 76:798-804.

25. Donovan JL, Crespy V, Oliveira M, Cooper KA, Gibson BB, Williamson G: (+)-Catechin is more bioavailable than (-)-catechin: Relevance to the bioavailability of catechin from cocoa. Free Rad Res 2006, 40:1029-1034.

26. Roelofsen PA: Fermentation, drying and storage of cacao beans. Adv Food Res 1958, 8:225-296.

27. Bracco U, Graihle N, Rostagno W, Egli RH: Analytical evaluation of cocoa curing in the Ivory Coast. J Sci Food Agric 1969, 20:713-716.

28. Kim H, Keeney PG: (-)-Epicatechin content in fermented and unfermented cocoa beans. J Food Sci 1984, 49:1090-1092.

29. Stahl LA, Miller KB, Apgar J, Sweigart DS, Stuart DA, Mchale N, Ou B, Kondo M, Hurst WJ: The preservation of cocoa antioxidant activity, total polyphenols, flavan-3-ols and procyanidin content in cooked and baked foods prepared with cocoa powder. J Food Sci 2009, 74:456-461. 
30. Robinson T, Ranalli AW, Phillips AW: Changes in cocoa tannins during processing. J Agric Food Chem 1961, 9:295-298.

31. Payne MJ, Hurst WJ, Miller KB, Rank C, Stuart DA: Impact of fermentation, drying, roasting and Dutch processing on epicatechin and catechin content of cacao beans and cocoa ingredients. J Agric Food Chem 2010, 58:10518-10527.

32. Gotti R, Furlanetto S, Pionzauti S, Cavrini V: Analysis of catechins in Theobroma cacao beans by cyclodextrin-modified micellar electrokinetic chromatography. J Chromatogr 2006, 1112:345-352.

33. Kofink M, Papagiannopoulos M, Galensa R: (-)-Catechin in cocoa and chocolate: Occurrence and analysis of an atypical flavan-3-ol enantiomer. Molecules 2007, 12:1274-1288.

34. Caligiani A, Cirlini M, Palla G, Ravaglia R, Arlorio M: GC-MS detection of chiral markers of cocoa beans of different quality and geographic origin. Chirality 2007, 19:329-334.

35. Nelson BC, Sharpless KE: Quantification of the predominant monomeric catechins in baking chocolate standard reference material by LC/APCIMS. J Agric Food Chem 2003, 51:531-537.

36. Gourieva KB, Tserevitinov OB: Method of evaluating the degree of fermentation of cocoa beans. USSR Patent No. 646 1979, 254.

37. Thompson SS, Miller KB, Lopez AS: Cocoa and Coffee. In Food Microbiology Fundamentals and Frontiers. 3 edition. Edited by: Doyle MP, Beuchar LR. American Society of Microbiology Press: Washington, DC; 2007:837-850

38. MARS Incorporated. MARS Botanical: CocoaPro. 2010 [http://cirkuhealth. com/Cocoa-101/CocoaPro.aspx]

39. Barry Callebaut Group. Acticoa. [http://www.acticoa.com/en].

40. Beckett ST: Industrial chocolate manufacture and use. Blackie, London van Nostrand Reinhold, NY; 1988, 388.

41. Minifie BW: Chocolate, cocoa and confectionery: Science and technology. van Nostrand Reinhold, NY; 3 1989, 904.

doi:10.1186/1752-153X-5-53

Cite this article as: Hurst et al: Impact of fermentation, drying, roasting and Dutch processing on flavan-3-ol stereochemistry in cacao beans and cocoa ingredients. Chemistry Central Journal 2011 5:53.

Publish with ChemistryCentral and every
scientist can read your work free of charge
"Open access provides opportunities to our
colleagues in other parts of the globe, by allowing
anyone to view the content free of charge."
W. Jeffery Hurst, The Hershey Company.
- available free of charge to the entire scientific community
- peer reviewed and published immediately upon acceptance
- cited in PubMed and archived on PubMed Central
- yours - you keep the copyright
Submit your manuscript here:
http:/lwww.chemistrycentral.com/manuscriptt

